\title{
\#SLOPCAB: Is the party over?
}

\author{
Craig R. Smith, MD
}

See related article on pages 1812-9.

The subanalysis ${ }^{1}$ from the Danish On-pump versus Offpump Randomization Study (DOORS) shows significantly lower graft patency after off-pump coronary artery bypass (OPCAB) versus on-pump coronary artery bypass, as assessed by angiography 6 months postoperatively ( $86 \%$ of grafts open on-pump vs $79 \%$ open off-pump, $P=.01$ ). Of note, equivalent intraoperative heparinization protocols were used. Patients undergoing $\mathrm{OPCAB}$ also received fewer total grafts in all territories, and twice as many patients undergoing OPCAB received fewer grafts than planned. Taken at face value, this is more bad news for OPCAB advocates! The only good news is that intracoronary shunts appear benign. Before quibbling over the details, readers of every bias should stand and applaud the authors for having the energy and tenacity to attempt a randomized trial on this complex clinical comparison.

Even the best clinical trial has vulnerabilities. In this one, angiography in only $57 \%$ of patients is a serious shortcoming. Defense of their conclusions requires a serious attempt by the authors to analyze the propensity for receiving follow-up angiography, with inclusion of key perioperative variables (eg, smoking, diabetes, postoperative complications, number of grafts, territories grafted, degree of proximal stenosis, and conduit selection), cumulative mortality as a competing variable to determine the balance of graftocclusion risk factors between cohorts, and whether the cohorts had equivalent propensity to undergo angiography. The authors have thoroughly and thoughtfully undertaken such an analysis, to the satisfaction of the Journal's fastidious statistical reviewers. They conclude that the propensity to undergo follow-up angiography, although not perfectly balanced, did not affect the overall results or conclusions.

To quibble over the details, it is worth nothing that the minimum requirement for "experience" with OPCAB

\footnotetext{
From the Columbia University College of Physicians and Surgeons, New York Presbyterian Hospital, New York, NY.

Disclosures: Author has nothing to disclose with regard to commercial support.

Received for publication Sept 18, 2014; accepted for publication Sept 19, 2014.

Address for reprints: Craig R. Smith, MD, Columbia University College of Physicians and Surgeons, New York Presbyterian Hospital, Milstein Building 7-435, Columbia University Medical Center, 177 Fort Washington Ave, New York, NY 10032 (E-mail: crs2@cumc.columbia.edu).

J Thorac Cardiovasc Surg 2014;148:1788-9

0022-5223/\$36.00

Copyright (c) 2014 by The American Association for Thoracic Surgery

http://dx.doi.org/10.1016/j.jtcvs.2014.09.054
}

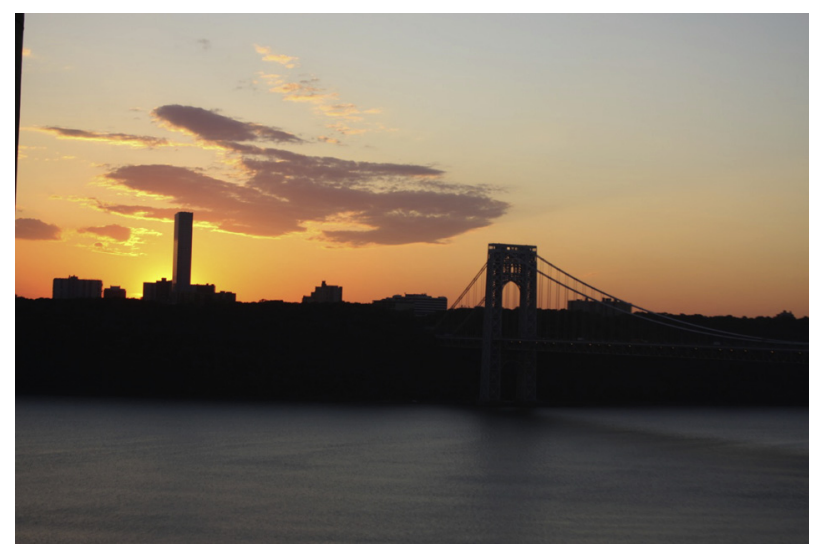

FIGURE 1.

was 25 cases that included circumflex grafting. This might qualify the technically gifted for the junior varsity, but it is a long way from a varsity letter. However, the authors point out that 10 of 12 surgeons in the trial had more than 100 cases, which is reasonably reassuring. In a similar vein, it could be argued that the entire experience reported in the DOORS is light on bilateral internal thoracic artery use, suggesting something less than cutting-edge coronary surgery. The authors have defended their experience threshold, and the predominance of left internal thoracic artery plus saphenous vein, with the argument that it more accurately portrays "real world" practice than would a trial generated by a pure collection of super-experts. This is undoubtedly true but obstructs a best-case argument for OPCAB, which already suffers from being hard to learn and to do, and is easily outshone by on-pump with respect to the number of highly skilled operators available for trials.

Equivalent heparinization, combined with reasonably skilled OPCAB surgeons, should focus attention on the technical issues that separate on-pump from off-pump, and the most important of those is the quality of the distal anastomosis. The hazard to the proximal anastomosis and "shaft" of a graft should be similar. A total of 121 of 250 occluded grafts involved the proximal anastomosis, versus 65 "shaft" and 96 distal anastomoses- $74 \%$ of the problems were seen in sites that might not be expected to differ greatly between the 2 techniques. The authors have not compared the frequency of anastomotic problems in the 2 groups by site. Although it is likely that the comparison would lack statistical power, imbalances between cohorts might support or undermine the conclusions.

Try as I might to find weaknesses in the DOORS data, I conclude that we cannot ignore this latest piece of evidence that one price paid for the technical difficulty of OPCAB is 
more frequent early graft failure, irrespective of heparinization. Even more sobering is the recent report from Kim and colleagues, ${ }^{2}$ who found a significant long-term survival advantage for on-pump in 5203 patients who were followed for a median of 6.4 years. This is particularly striking because $60 \%$ of coronary bypass surgeries in Korea have been done off-pump, and the authors are highly experienced by any metric.

Ironically, OPCAB was inspired 20 years ago by a desire to compete with percutaneous coronary intervention (PCI) by offering a less-invasive alternative. Yet now, when compared with on-pump, OPCAB is behaving more and more like PCI in multivessel disease or diabetes-except that it lacks any of the obvious benefits of a procedure that is truly less invasive. I say this from the perspective of a surgeon who has been doing more than $80 \%$ of his coronary procedures off-pump! I flatter myself that I have become very proficient, and that I know who should be in the $20 \%$, but the steady drumbeat of opposing data is becoming hard to tune out. There are undeniably instances where OPCAB can be crucial-the porcelain aorta, for example. But maintaining proficiency for those cases may be hard to justify, because complex PCI usually will be a reasonable alternative.

\section{References}

1. Houlind K, Fenger-Grøn M, Holme SJ, Kjeldsen BJ, Madsen SN, Rasmussen BS, et al, for the DOORS Study Group. Graft patency after off-pump coronary artery bypass surgery is inferior even with identical heparinization protocols. Results from the Danish On-pump Versus Off-pump Randomization Study (DOORS). $J$ Thorac Cardiovasc Surg. 2014;148:1812-9.

2. Kim JB, Yun SC, Lim JW, Hwang SK, Jung SH, Song H, et al. Long-term survival following coronary artery bypass grafting. J Am Coll Cardiol. 2014;63: 2280-8. 